\title{
PARALLEL PLATE WAVEGUIDE WITH AMC-PEC-AMC STRIPS
}

\author{
P. Padilla de la Torre ${ }^{(1)}$, J.M Fernández ${ }^{(1)}$, M. Sierra-Castañer ${ }^{(1)}$ \\ ${ }^{(1)}$ Universidad Politécnica de Madrid, ETSI Telecomunicación C-415., 28040 Madrid (Spain), Email: \\ ppadilla00@gr.ssr.upm.es
}

\begin{abstract}
The aim of this paper is to demonstrate that it is possible to perform a waveguide using parallel plates and some metamaterial surfaces. The lack of lateral metallic walls in the waveguide is replaced by the effect of these metamaterials. An AMC-PEC-AMC structure is designed, simulated and prototyped. The advantage of this structure is due to the planar architecture and its low cost.

In this paper, we use the structure described as if it were the feeding part of a planar slot antenna, in order to demonstrate the chance of using this structures in this antennas. Reflection and radiation pattern measurements are presented.
\end{abstract}

\section{INTRODUCTION}

The paper below summarizes a complete research work to obtain a multilayer dielectric and metallic structure between parallel plates, working as if the whole structure were a conventional waveguide. The waveguide effect is achieved by using some printed surfaces with an artificial magnetic conductor behaviour (AMC) that replace the conducting walls. In that way, the manufacture complexity is drastically decreased, as it is only needed planar architecture. As a direct application of this structure, it could be used as the feeding stage of a planar antenna.

\section{VIRTUAL WAVEGUIDE}

Artificial magnetic conductors have, among others, an specific feature that make them extremely useful in our design: Over an AMC surface, the tangential magnetic field tends to be null $\left(\mathrm{H}_{\mathrm{t}}=0\right)$.

In order to obtain materials with this artificial magnetic conductor behaviour, it is necessary to use planar architecture that implies Frequency Selective Surfaces (FSS). One particular design is the UC-EBG structure [3] (Uniplanar Compact EBG), that, as it is shown in Fig. 1. The periodic spatial repetition of an UC-EBG structure let us design surfaces with a AMC behaviour.

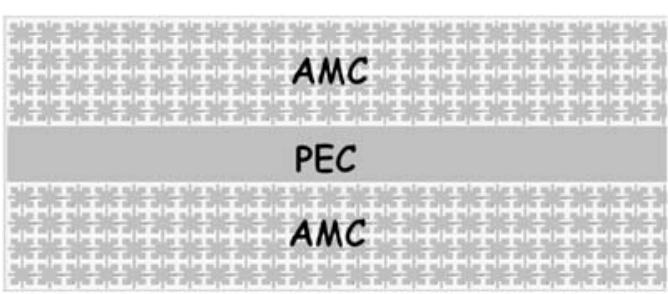

Figure. 1. considered AMC-PEC-AMC structure.

As it is seen in Fig. 1, a copper strip is going to be placed, surrounded by AMC behaviour strips. In our model, the metallic strip is equivalent to the base side of a conventional waveguide, while the AMC surfaces tend to work as vertical metallic walls of the waveguide. To conclude the virtual waveguide, we place another identical structure in front of the previous one. Fig. 2 present clearly our model and the equivalent one. More information could be found in [1].

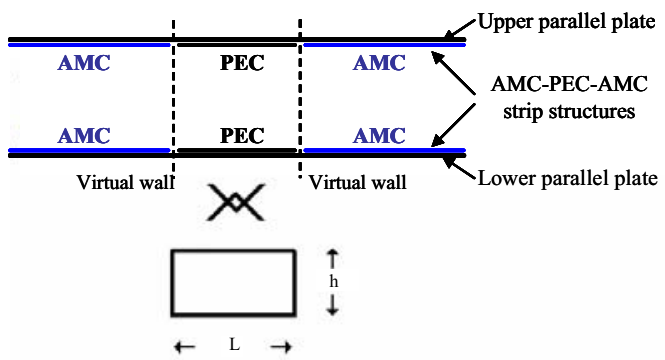

Figure. 2. AMC-PEC-AMC structure and its equivalent model.

\section{SIMULATIONS}

To prove that the structure described works properly, it is necessary to simulate a model with simulation software.

The software applied allow the user to assign specific ideal properties to the faces of different surfaces. This facility is used to generate an ideal AMC-PEC-AMC structure. Later, the real model is going to be simulated. In both cases, it is going to be considered the values below:
Working frequency $\approx 12.7 \mathrm{GHz}$.

Thickness between parallel plates: $9.5 \mathrm{~mm}$.

PEC width: $19 \mathrm{~mm}$.

AMC structure periodicity: 37 x 3 . 


\subsection{Ideal structure simulation}

The two AMC strips are replaced by ideal ones, defined with the $\mathrm{H}_{\mathrm{t}}=0$ property over their surface, as it is shown in Fig. 3a.

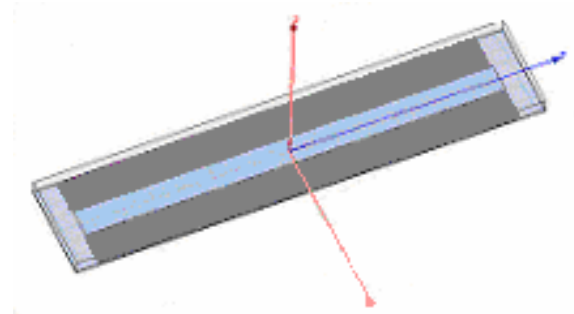

(a)

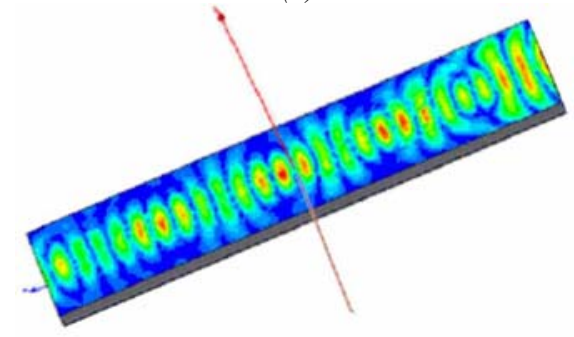

(b)

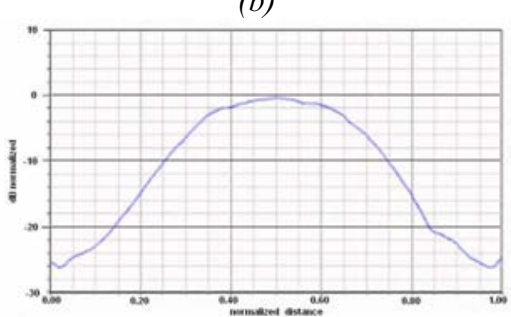

(c)

Figure. 3. (a) Ideal AMC-PEC-AMC structure. Electric field distribution (b) over the ideal AMC-PEC-AMC structure, (c) in a ideal AMC-PEC-AMC cut plane.

Fig. $3 \mathrm{~b}$ shows the electric field distribution when the structure is excited with a port in one edge ( $\mathrm{z}$ propagation direction). In the same way, in Fig. 3c, the module of the field distribution in one cut plane (xy plane) is obtained.

\subsection{Real structure simulation}

Original AMC strips are placed where the ideal strips were (Fig. 4a) and the new structure is simulated with CST 5.0. In the same way, it is possible to get the field distribution when exciting with a port at the edge, working at $12.65 \mathrm{GHz}$ (Fig. 4b) and $12.95 \mathrm{GHz}$. It can be noticed that at other frequencies different from the working ones (Eg.12GHz) the wave does not propagate (Fig. 4c).

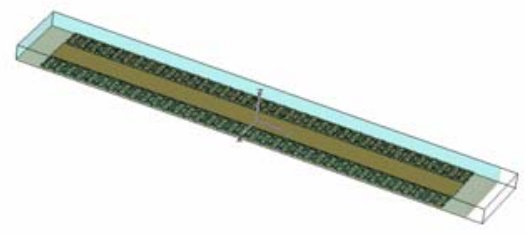

(a)

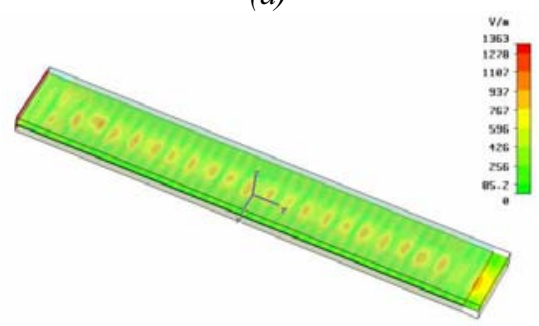

(b)

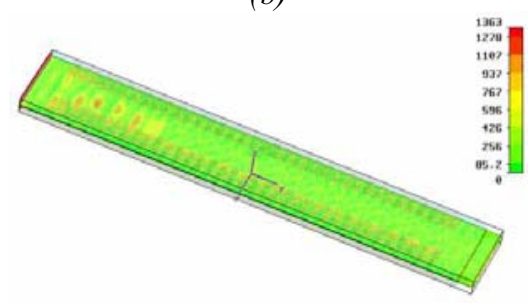

(c)

Figure. 4. (a) Real AMC-PEC-AMC structure. Electric field distribution in this structure: (b) one working frequency $(12.65 \mathrm{GHz})$; (c) different frequency.

\section{IMPLEMENTATION AND PROTOTYPES}

In the implementation process, two structures are manufactured to analyze and validate the simulation results, as it is presented in Fig. 5. The first of them (Fig. 5a) is quite important in order to validate the theoretical and simulated results. For this reason, in this paper almost all the efforts are going to be placed in this first structure.

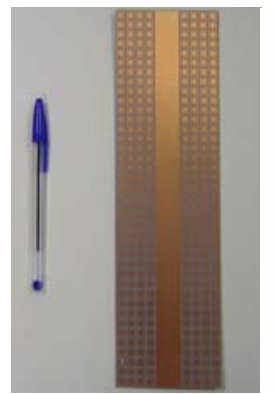

(a)

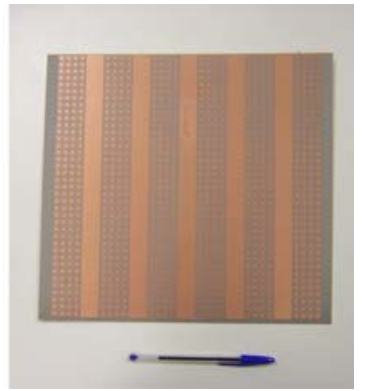

(b)
Figure. 5. (a) AMC-PEC-AMC prototype. (b) Periodic prototype of (a). 
Among the different ways of measuring the effect enabling or disabling the propagation, those which better show the effects and are able to be performed are presented.

\subsection{Prototype 1. Coaxial excitation analysis}

The aim of the present measurement is trying to show the effect of wave guidance, allowing the propagation over the PEC zone, disabling it over the AMC zones. In this assembly, it is supposed to maintain the dimensions used in the previous simulation step; in that way the structure is located between two parallel plates, excited with a coaxial excitation, analyzing the field in a $\lambda / 2$ ring surrounding the excitation point. The test scheme is presented in Fig. 6.

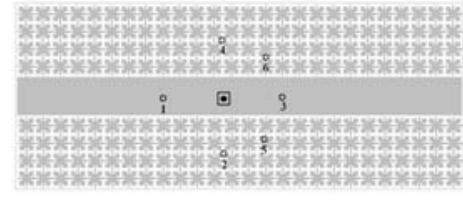

(a)

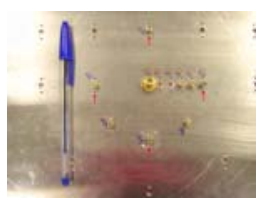

(b)
Figure. 6. (a) AMC-PEC-AMC structure analysis with coaxial excitation. (b) Measure prototype.

The use of a vectorial network analyzer help us to verify that the excitation is clearly matched and to obtain the electric field amplitude in the different points of the ring, in a wide frequency range (12GHz-13.45GHZ). The best results appear at $12.65 \mathrm{GHz}$ and $12.95 \mathrm{GHz}$. In order to represent the results in a suitable way, two graphs are prepared and displayed (Fig. 7): for the 'cardinal points' (1, 2, 3 and 4 points) and for the semicircumference $(2,5,3,6$ and 4 point), for both frequencies.

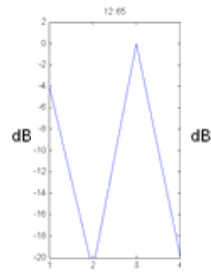

(a1)

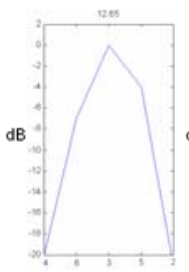

(a2)

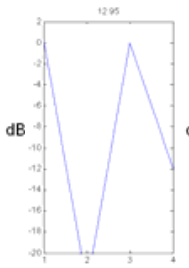

(b1)

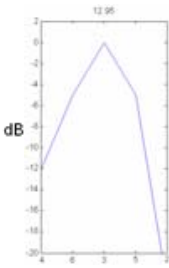

(b2)
Figure. 7. Electric field distribution at the 'cardinal points'(a1\&b1) and in the semicircumference (a2\&b2). (a) $12.65 \mathrm{GHz}$. (b) $12.95 \mathrm{GHz}$.

As it is seen in 'cardinal points' in both figures, points 1 and 3 (over PEC) present maximums of transmission, while points 2 and 4 present minimums (over AMC), with a difference of $20 \mathrm{~dB}$.

\subsection{Prototype 1. Uniform excitation analysis}

In addition to the previous analysis another test could be done to assure the right operation of the structure. A line is located over the structure without electrical contact with the AMC-PEC-AMC plane, so that it generates a uniform wave in amplitude propagating throughout the plane, as shown in Fig. 8. Points in a parallel line to the line of excitation is analyzed for different distances from the excitation.

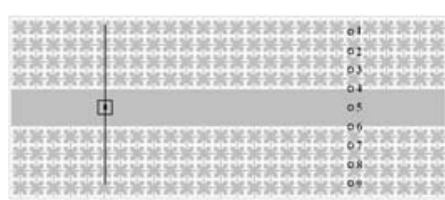

(a)

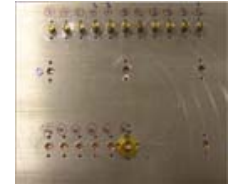

(b)
Figure. 8. (a) AMC-PEC-AMC structure analysis considering uniform excitation. (b) Measure Prototype.

At the working frequencies, the field configuration is shown in Fig. 9. These results are quite similar to those obtained in simulations, as it was expected.
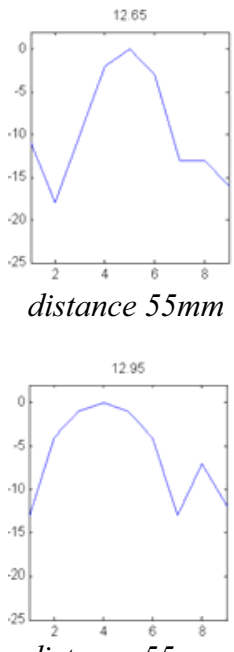

distance $55 \mathrm{~mm}$

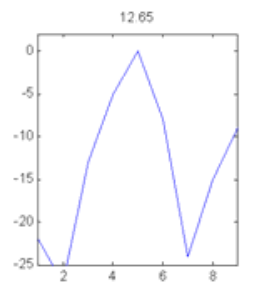

distance $110 \mathrm{~mm}$

(a)

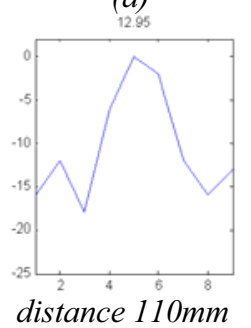

(b)

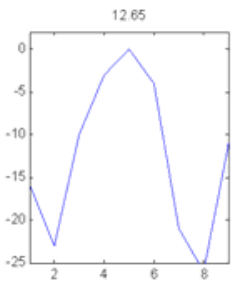

distance $165 \mathrm{~mm}$

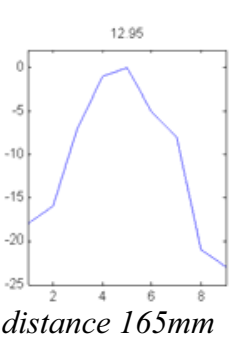

distance $165 \mathrm{~mm}$
Figure. 9. Section field distribution, (uniform excitation). (a) $12.65 \mathrm{GHz}$ (b) $12.95 \mathrm{GHz}$

\subsection{Prototype 2.}

At last, measurements of the prototype 2 are presented. Due to the excitation (which is a slot array) and its narrow band width, measurements are only related to $12.65 \mathrm{GHz}$ (one of the working frequencies). Measured results show a proper behaviour (Fig. 10b). 


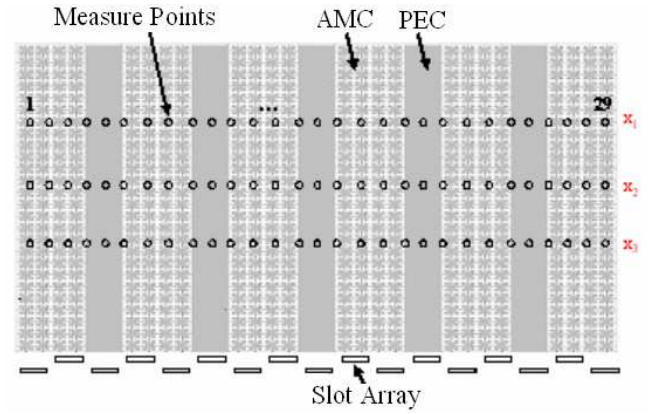

(a)

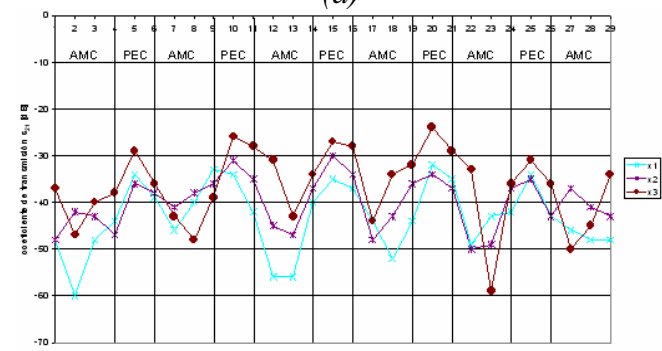

(b)

Figure. 10 (a) Measurement scheme. (b) Electric field distribution at $12.65 \mathrm{GHz}$

\section{ANTENNA DESIGN}

In order to validate al the previous results it is decided to design a quite simple planar antenna, in which our virtual waveguide works like the feeding stage. One of the easiest models that can be thought about is a slot array excited by the structure in figure 5.a, as it is seen in Fig. 11.

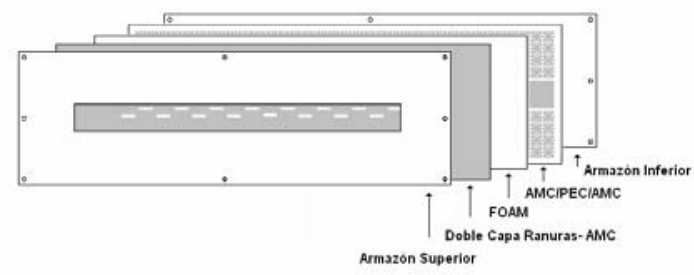

Figure. 11-Antenna assembly scheme.

The value of the effective wavelength inside the waveguide $\left(\lambda_{\mathrm{g}}\right)$ is fundamental when designing the slot array. Therefore, it is necessary to recover the simulation results of the first prototype, as it is shown in Fig. 12.
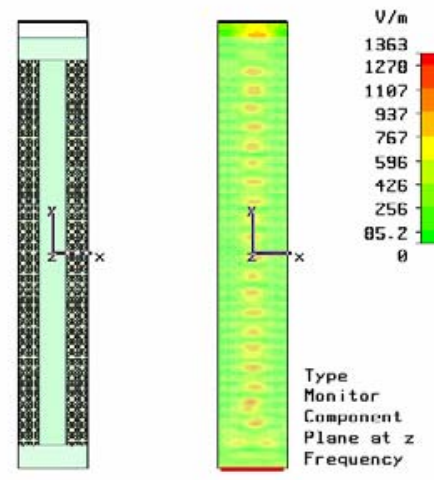

Figure. 12. Field distribution inside the first prototype at $12.65 \mathrm{GHz}$.

(CST 5 simulation).

Fig. 13 shows the manufactured antenna, after the assembly of the different parts.

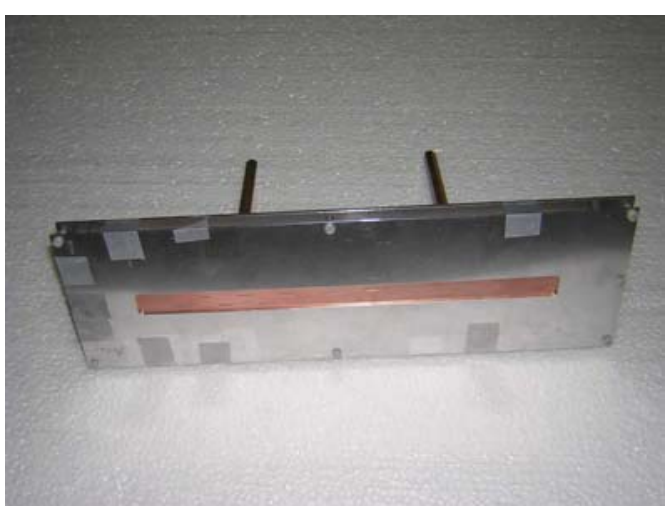

Figure.13. Complete antenna.

The reflection measurements are presented in Fig. 14. At one of the working frequencies $(12.65 \mathrm{GHz})$ the reflection is less than $-20 \mathrm{~dB}$.

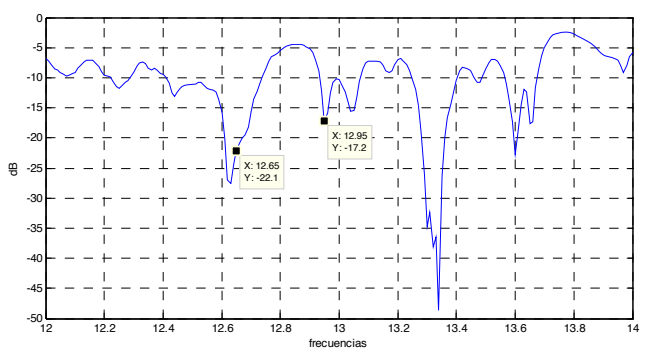

Figure. 14. Antenna $S_{11}, \lambda_{g}=26.5 \mathrm{~mm}$.

Finally, the radiation pattern is measured in an anechoic chamber, at $12.65 \mathrm{GHz}$ (Fig. $15 \mathrm{y} \mathrm{16}$ ). 


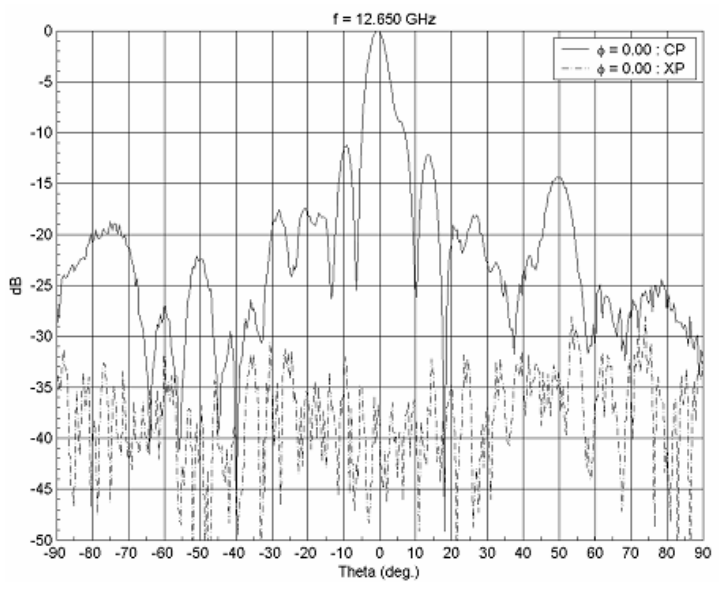

Figure.15. Horizontal radiation pattern (array).

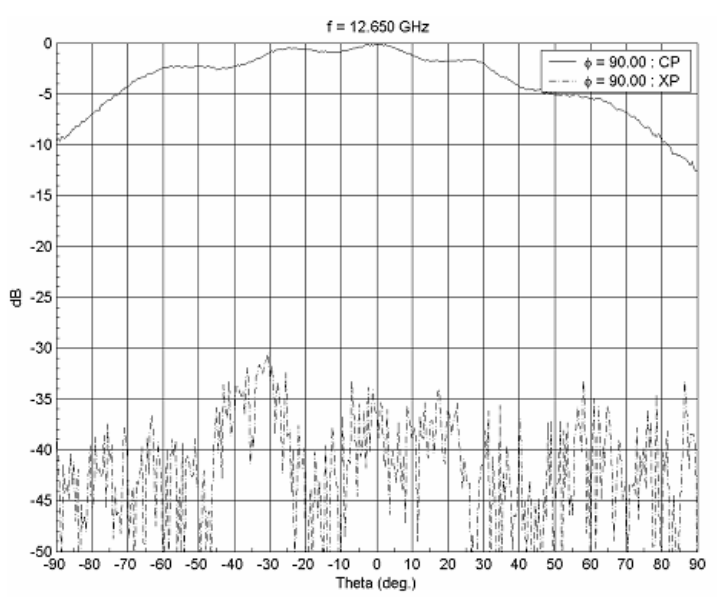

Figure.16. Vertical radiation pattern (slot).

As it is noticed, the radiation pattern points to $0^{\circ}$, what means that the $\lambda_{\mathrm{g}}$ value was properly taken. Although the radiation pattern has to be improved, it demonstrates the proper operation of an antenna with virtual waveguide.

\section{CONCLUSIONS}

Best efforts have been made in order to assure that the structure works in a proper way. For this reason, this paper includes some software simulations and the prototype measurement and evaluation. This work demonstrates the feasibility of applying the AMC-PECAMC configuration in parallel plate waveguides to enhance the properties of the wave propagation. In the case of using several AMC-PEC-AMC structures working like consecutive virtual waveguides in a plane, we reduce the coupling between each other. The results obtained let us consider different possibilities using these architecture to perform planar antennas with specific features.

\section{ACKNOWLEDGEMENT}

The simulations above have been realized using CST Microwave Studio 5.0 under co-operation agreement between CST and Polytechnic University of Madrid. NY substrate used in the prototypes was kindly given by NELTEC S.A.

\section{REFERENCES}

[1] J.M. Fernández, P. Padilla de la Torre, M. Sierra Castañer. "Artificial Magnetic Conductors enhancing the wave propagation in Oversized Parallel Plate Waveguides for Planar Antenna Applications". Proceedings of the European Microwave Association (ProcEuMa). Marzo 2006.

[2] P. Padilla de la Torre, J.M. Fernández, M. Sierra Castañer "Tiras AMC-PEC-AMC en Guías de Onda de Placas Paralelas". XX Simposium Nacional de la Unión Científica Internacional de Radio (URSI) Septiembre 2005, Gandía, España.

[3] Douglas J. Kern, Douglas H. Werner, A. Monorchio and L. Lanuzza. "The Design Synthesis of Multiband Artificial Magnetic Conductors Using High Impedance Frecuency Selective surfaces". IEEE trans. Antenas and Propagation, vol. 53 no. 1,Jan. 2005.

[4] A. Aminian, F. Yang, and Yahya Rahmat-Samii, "In-phase reflection and EM wave suppression characteristics of electromagnetic band gap ground planes," IEEE Antennas and Propagation Society International Symposium, Ohio, 2003.

[5] S. Maci, Per-Simon Kildal. "Hard and Soft Surfaces Realized by FSS Printed on a Grounded Dielectric Slab" IEEE, 2004.

[6] Y.J. Park, A. Herschlein and W. Wiesbeck. "A Photonic Bandgap (PBG) Structure for Guiding and Suprissing Surface waves in millimeter-wave antennas". IEEE Trans. Microwave Theory, vol. 49 no. 10 , Oct. 2001.

[7] P.-S. Kildal, "Artificially soft and hard surfaces in electromagnetics,".IEEE Trans. Antennas Propagat., vol. 38, pp. 1537-1544, Oct. 1990

[8] J. Anders "Properties of Waves Guided Between Parllel, Artificially Hard Surfaces". IEEE, 1993. 